\title{
El estado nutricional y su relación con la actividad física, el nivel socioeconómico y el rendimiento académico
}

Karin Jacqueline Milla, Renata Marcela Valle ${ }^{1}$

\section{RESUMEN}

Objetivo: Analizar la relación entre el estado nutricional con la actividad física, nivel socioeconómico y rendimiento académico de los alumnos de la carrera de Química y Farmacia de la UNAH en el tercer período 2014. Método: Estudio correlacional y transversal, de una muestra de 198 alumnos, de edades entre 17 a 30 años. Datos recolectados mediante cuestionarios validados de: datos generales, medidas antropométricas, recordatorio de 24 horas, índice global, nivel de actividad física y socioeconómica. En las pruebas el nivel de significación empleado fue de 0.01. Resultados: Según el Índice de masa corporal (IMC) el 13\% tiene peso insuficiente, $20.2 \%$ sobrepeso y $7.6 \%$ obesidad, de acuerdo al índice de cintura cadera (ICC) el $41 \%$ tienen riesgo de enfermedades crónicas no transmisibles (ECNT), los varones mostraron mayor grasa corporal, no presentan las diferencias propias de los patrones según el sexo. La dieta no es balanceada, es monótona, alta en proteínas, carbohidratos, baja en grasas, consumo de pescados, mariscos, frutas y verduras. La ingesta hídrica es menor a lo recomendado en $80 \%$ de los alumnos, $44 \%$ ingiere 4 comidas al día. El balance energético es negativo (79.3\%) y la distribución calórica es inadecuada. Las pruebas bioquímicas reflejan un buen estado nutricional, el $44 \%$ presentan un valor menor de lo normal de lipoproteínas de alta densidad (HDL). La actividad física fue ligera en el $70 \%$ de los alumnos. El rendimiento académico es bueno en $77 \%$ y el nivel socioeconómico es medio en $57 \%$. Conclusión: No se encontró diferencias estadísticamente significativas entre las variables.

Palabras clave: estado nutricional, actividad física, nivel socioeconómico, rendimiento académico.

\footnotetext{
${ }^{1}$ Beneficiarias de una beca básica de la DICYP. Grupo de Investigación Análisis Químico. Profesoras del Departamento de Química, Facultad de Ciencias Químicas y Farmacia. UNAH: jackiemilla61@yahoo.com; analítica_iv@yahoo.com
} 


\section{ABSTRACT}

Objective: To analyze the relationship between nutritional status and physical activity, socioeconomic status and academic performance of the third period Chemistry and Farmacy students in 2014. Method: Correlational and cross-sectional study of a sample of 198 students, ages 17 to 30 years. Data collected through validated questionnaires of: general information, anthropometric measurements, 24-hour reminder, global index, level of physical and socioeconomic activity. In the tests, the level of significance used was 0.01 . Results: According to the body mass index (BMI) 13\% have insufficient weight, $20.2 \%$ are overweight and $7.6 \%$ are suffering from obesity. According to the hip waist index (CCl), $41 \%$ have risk of chronic noncommunicable diseases (CNCD), males showed higher body fat, and don't present pattern differences according to sex. The diet is not balanced, high in protein \& carbohydrates, low in fat, consumption of fish, seafood, fruits \& vegetables. The water intake is less than what is recommended. In $80 \%$ of the students, $44 \%$ eat 4 meals a day. In $79.3 \%$, the energy balance is negative and the caloric distribution is inadequate. The biochemical tests reflect a good nutritional status, $44 \%$ have a lower than normal value of high density lipoproteins (HDL). Physical activity was minimum in $70 \%$ of the students. In $77 \%$ of the students, the academic performance is good and in $57 \%$ the socioeconomic level is average . Conclusion: No statistically significant differences were found between the variables.

Keywords: nutritional status, physical activity, socioeconomic status, academic performance. 


\section{INTRODUCCIÓN}

En la actualidad, datos estadísticos de la Organización Mundial de la Salud (OMS, 2009) revelan que las enfermedades crónicas no transmisibles (ECNT) provocan el $75 \%$ de las muertes a nivel mundial, en Honduras el $69 \%$ de las muertes, afirma que se deben a una mundialización de hábitos poco saludables, como las dietas malsanas y la inactividad física que conllevan a una tendencia de exceso de grasa corporal donde prevalece a nivel mundial sobrepeso en los hombres y obesidad en las mujeres, además muchos estudios a nivel de Latinoamérica (Argentina, Brasil, Colombia, Cuba, Ecuador, El Salvador, México, Venezuela, Guatemala) lo confirman según la (OMS, 2009).

Estudios diversos de la Organización Panamericana de la Salud (OPS)-OMS realizados en 2009 muestran que las condiciones socioeconómicas de las familias inciden sobre el estado de la salud y la nutrición, el nivel socioeconómico de las familias, es una variable estadísticamente significativa que explica la inequidad en los indicadores de salud y nutrición (Carbajal, Azcona, A, 2013). Además, se conoce que la salud y la educación son unas de las principales fuentes de bienestar de la sociedad, factor fundamental para el desarrollo socio-cultural y económico de una nación (Álvarez, L.S. etal, 2012) (Cordoba, D.etal, 2013).

Hay suficiente evidencia disponible de la relación que existe entre el modelo de consumo alimentario y algunas enfermedades crónico-degenerativas (Carbajal, Azcona, A, 2013), también se conoce que la efectividad del trabajo intelectual depende de lo que hemos consumido en la alimentación (Gallardo. Wangi B.A. 2011). Por ende, la nutrición incide en la salud, conocer la ingesta de alimentos de la dieta de acuerdo a las necesidades fisiológicas del organismo permite ajustar el aporte de nutrientes por medio de dietas equilibradas.

La iniciativa de realizar este estudio surge de la problemática del consumo inadecuado de alimentos acompañado de la inactividad física, trastornos en el comportamiento alimentario (Carbajal, Azcona, A, 2013) de los jóvenes universitarios y de la evidencia de la relación que existe entre el modelo de consumo alimentario y el rendimiento académico de los alumnos9. Asimismo, es de suma importancia la intervención en esta época universitaria ya que pronto conformarán la población económicamente activa y productiva del país, pues existen evidencias de que los hábitos alimentarios son modificables y también se conoce que la prevención es la mejor alternativa (Rivera M.F. 2010). Además, para disminuir la prevalencia de las enfermedades crónicas 
no transmisibles en la población universitaria como la carga económica que estas representan para el país y por último es un estudio novedoso ya que nunca se ha realizado en la facultad de Ciencias Químicas y Farmacia de la UNAH.

Se encontró que los estudios sobre el estado nutricional en Honduras se han realizado en su mayoría en niños (Rivera M.F. 2010), mujeres embarazadas y adultos mayores (Aguirre, Cando, 2012)pero no en jóvenes o adolescentes pues se supone que este grupo de la población tiene buena salud.

Hace diez años en la UNAH el riesgo académico (alumnos reprobados, insuficientes) era del $40 \%$ actualmente ha disminuido a un $12 \%$, sin embargo, en la Carrera de Química y Farmacia es del $32 \%$, por ello se quiere conocer el estado nutricional de los alumnos y si este incide en su rendimiento académico.

El objetivo de este estudio fue evaluar el estado nutricional de los alumnos de la carrera de Química y Farmacia por medio de parámetros antropométricos (IMC, ICC y $\%$ Grasa), dietéticos ( $\%$ proteína, $\%$ carbohidratos, $\%$ grasa total, número comidas, ingesta calórica, ingesta hídrica, balance energético) y pruebas bioquímicas (hemograma, glucosa, colesterol, lipoproteínas de alta densidad (HDL), lipoproteínas de baja densidad (LDL), triglicéridos, albúmina y ácido úrico) para establecer su relación con el rendimiento académico, la actividad física y el nivel socioeconómico.

\section{MÉTODOS}

Este estudio es cuantitativo, no experimental, correlacional y transversal, en él se investigó la relación entre el estado nutricional, la actividad física, el rendimiento académico y el nivel socioeconómico de 198 alumnos de la carrera de Química y Farmacia ( $78.8 \%$ mujeres y el $21.2 \%$ varones). Se les solicitó firmar consentimiento informado a quienes deseaban participar voluntariamente, muestreo intencional (Arias Fidias, G, 2012). Y fueron debidamente instruidos, garantizándoles condiciones de intimidad y confidencialidad de la información proporcionada y generada.

Valoración antropométrica (Esenano Aquilar, etal. 2012) se tomaron las medidas de peso y talla por triplicado con una balanza digital de pared con tallímetro, Seca Modelo 769132211994 para determinar el IMC, \% de grasa y ICC.

Valoración dietética (mataix Verdri, J, 2009) se usó el registro de recordatorio de 24 
horas validado, para cuatro días de una semana incluyendo un día del fin de semana. El consumo de alimentos se transformó en energía y macronutrientes empleando las tablas de composición de los alimentos para Centroamérica y Panamá del INCAP17 valorándose la adecuación del aporte nutritivo.

Actividad física se valoró por una semana utilizando un cuestionario validado de actividades clasificadas en ligera, moderada e intensa.

Balance energético los requerimientos de energía se estimaron a partir de la fórmula predictiva de Harris-Benedict (1919) de acuerdo a la ingesta calórica y nivel de actividad física realizada.

Rendimiento académico: se registró el índice académico global de cada alumno.

Nivel socioeconómico: se determinó usando un cuestionario validado, conforme criterios del ingreso familiar, escolaridad de la madre, colonia donde habitan y si poseen vehículo

Tratamiento estadístico: El análisis estadístico se realizó mediante el programa informático SPSS versión 20 (IBM) con un nivel de significación de 0,01. Se empleó la correlación de Pearson.

\section{RESULTADOS}

El estado nutricional de los alumnos de la carrera de Química y Farmacia a través de la valoración del IMC (gráfico 1) se obtuvo que el $60 \%$ tienen normopeso, 13\% peso insuficiente y $20 \%$ sobrepeso y $7.6 \%$ de obesidad. De acuerdo al ICC el $49 \%$ de las alumnas y el $33 \%$ de los alumnos tienen riesgo de padecer enfermedades crónicas no transmisibles (tabla 1). Respecto al porcentaje de grasa corporal (tabla 1), los alumnos presentaron una mayor tendencia al exceso (31\%) que las alumnas $(21 \%)$, en consecuencia, no se presentan las diferencias propias de los patrones según el sexo (Carbajal, Azcona, A, 2013). 
Gráfico 1. El índice de masa corporal de los alumnos

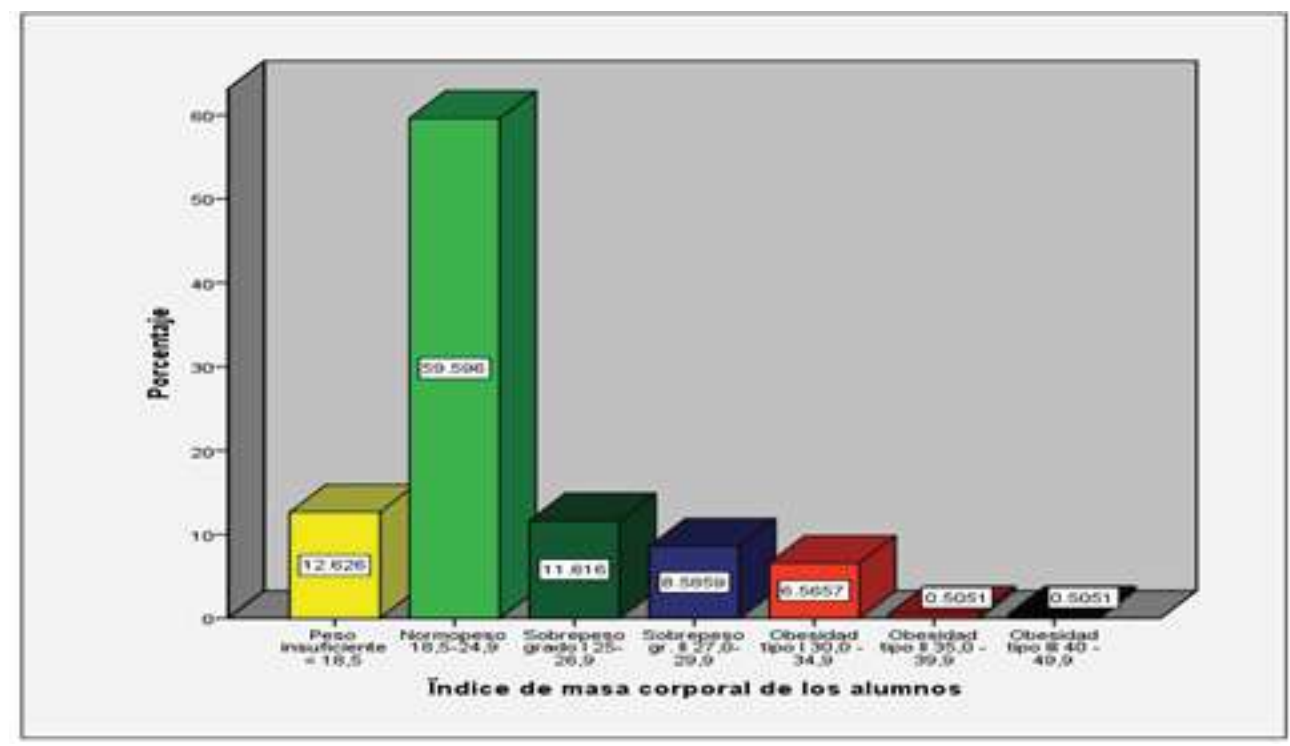

Fuente: elaboración propia a partir del registro de datos antropométricos

Tabla 1. Porcentaje de grasa corporal e índice cintura cadera de alumnos y alumnas

\begin{tabular}{|c|c|c|c|c|}
\hline & $\begin{array}{l}\text { Indicadores } \\
\text { alumnos }\end{array}$ & $\begin{array}{c}\text { Porcentaje } \\
\text { alumnos }\end{array}$ & $\begin{array}{l}\text { Indicadores } \\
\text { alumnas }\end{array}$ & $\begin{array}{c}\text { Porcentaje } \\
\text { alumnas }\end{array}$ \\
\hline \multirow{3}{*}{ 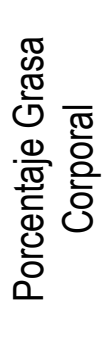 } & $\begin{array}{l}\text { Menor a lo normal } \\
\quad<10 \%\end{array}$ & 4.8 & $\begin{array}{c}\text { Menor a lo normal } \\
<20 \%\end{array}$ & 3.8 \\
\hline & $\begin{array}{l}\text { Normal } \\
10-20 \%\end{array}$ & 64.3 & $\begin{array}{c}\text { Normal } \\
20-30 \%\end{array}$ & 75.4 \\
\hline & $\begin{array}{c}\text { Mayor a lo normal } \\
>20 \%\end{array}$ & 31.0 & $\begin{array}{c}\text { Mayor a lo normal } \\
\quad>30 \%\end{array}$ & 20.9 \\
\hline \multirow{4}{*}{ 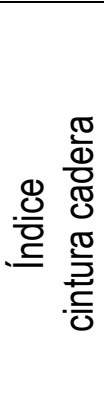 } & Sin riesgo $<0,83$ & 28 & Sin riesgo $<0,72$ & 50.6 \\
\hline & $\begin{array}{c}\text { Riesgo bajo } \\
0,83-0,88\end{array}$ & 7 & $\begin{array}{c}\text { Riesgo bajo } \\
0,72-0,78\end{array}$ & 19 \\
\hline & $\begin{array}{c}\text { Riesgo moderado } \\
0,88-0,95\end{array}$ & 6 & $\begin{array}{c}\text { Riesgo moderado } \\
0,78-0,82\end{array}$ & 28.5 \\
\hline & $\begin{array}{l}\text { Riesgo muy alto } \\
>1,01\end{array}$ & 1 & $\begin{array}{c}\text { Riesgo muy alto } \\
>0,82\end{array}$ & 1.9 \\
\hline
\end{tabular}

Fuente: Elaboración propia a partir del registro de datos antropométricos y resultados de \%GC e ICC 
Como resultado de la valoración dietética (tabla 2) de los alumnos no es balanceada, es monótona, alta en proteínas ( $74.2 \%$ de los alumnos), carbohidratos ( $45 \%$ de los alumnos) y baja en grasas ( $93.4 \%$ de los alumnos). Se caracteriza por bajo consumo de pescados, mariscos, frutas y verduras por todo lo anterior el patrón de alimentación está alterado por la disponibilidad de comidas de menor precio y alto contenido energético sustituyendo alimentos de superior valor nutritivo. La ingesta hídrica es menor a lo recomendado en $80 \%$ de los alumnos (tabla 2). El $26 \%$ ingiere 5 comidas al día, el $44 \%$ ingiere 4 comidas al día, el $26 \%$ ingiere 3 comidas al día. El balance energético es negativo (79.3\%) y la distribución calórica es inadecuada, en el desayuno la ingesta se cumple en $30 \%$ de la muestra, en el almuerzo en $23 \%$, en la cena $34 \%$ y en las meriendas sólo el $1 \%$ (tabla 2 ) y no consumen suplementos.

Tabla 2. Valoración dietética

\begin{tabular}{|c|c|c|c|c|}
\hline \multirow{2}{*}{\multicolumn{2}{|c|}{$\begin{array}{l}\text { INDICADORES } \\
\text { INCAP }\end{array}$}} & \multicolumn{3}{|c|}{ PORCENTAJE DE ALUMNOS } \\
\hline & & $\begin{array}{l}\text { MENOR A LO } \\
\text { RECOMENDO }\end{array}$ & $\begin{array}{c}\text { LO } \\
\text { RECOMENDADO }\end{array}$ & $\begin{array}{c}\text { MAYOR A LO } \\
\text { RECOMENDADO }\end{array}$ \\
\hline \multirow{3}{*}{ 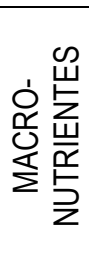 } & $\begin{array}{c}\text { Proteínas } \\
12 \%-15 \% 17\end{array}$ & $7.1 \%$ & $18.7 \%$ & $74.2 \%$ \\
\hline & $\begin{array}{c}\text { Carbohidratos } \\
55 \%-60 \%{ }^{17}\end{array}$ & $34.8 \%$ & $20.2 \%$ & $44.9 \%$ \\
\hline & $\begin{array}{c}\text { GRASAS } \\
30 \%-35 \%{ }^{9}\end{array}$ & $93.4 \%$ & $6.6 \%$ & $0 \%$ \\
\hline 迈 & $\begin{array}{c}\text { Ingesta hídrica } \\
1.5 \mathrm{~L}-2.0 \mathrm{~L}^{17}\end{array}$ & $80.3 \%$ & $16.2 \%$ & $3.5 \%$ \\
\hline \multirow{4}{*}{ 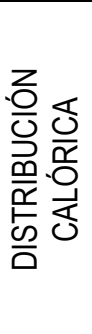 } & $\begin{array}{l}\text { Desayuno } \\
20 \%-25 \% 17\end{array}$ & $32.8 \%$ & $29.8 \%$ & $37.4 \%$ \\
\hline & $\begin{array}{c}\text { Meriendas } \\
20 \% 17\end{array}$ & $69.7 \%$ & $1.0 \%$ & $29.3 \%$ \\
\hline & $\begin{array}{c}\text { Almuerzo } \\
30 \%-35 \% 17\end{array}$ & $33.3 \%$ & $23.2 \%$ & $43.4 \%$ \\
\hline & $\begin{array}{c}\text { CENA } \\
20 \%-30 \% 17\end{array}$ & $20.2 \%$ & $33.8 \%$ & $46 \%$ \\
\hline
\end{tabular}

Fuente: Elaboración propia a partir recordatorio de 24 horas y tablas de ingestas recomendadas del INCAP 
Las pruebas bioquímicas (tabla 3) reflejan un buen estado nutricional, no se reportó ningún caso de anemia, así mismo el $96 \%$ de los alumnos tienen un perfil lipídico normal, el $85 \%$ de los alumnos presentaron la glucemia normal, el $87 \%$ de los alumnos el ácido úrico normal y el $81 \%$ de los alumnos valores normales de albúmina indicando un buen estado de salud en general, sin embargo, se encontró que el $44 \%$ de los alumnos presentan un valor menor a lo normal de lipoproteínas de alta densidad (HDL).

Tabla 3: Pruebas bioquímicas

\begin{tabular}{|l|c|c|c|}
\hline \multicolumn{1}{|c|}{ Indicadores } & \multicolumn{3}{|c|}{ Porcentaje de los alumnos } \\
\hline Química sanguínea & $\begin{array}{c}\text { MENOR A LO } \\
\text { NORMAL }\end{array}$ & $\begin{array}{c}\text { VALOR } \\
\text { NORMAL }\end{array}$ & $\begin{array}{c}\text { MAYOR A LO } \\
\text { NORMAL }\end{array}$ \\
\hline Glucosa 70 - 110 & $12.8 \%$ & $85.3 \%$ & $1.9 \%$ \\
\hline Ácido úrico 2.5-7.0 & $10.3 \%$ & $87.2 \%$ & $2.6 \%$ \\
\hline Albúmina 35 - 50 & $9.0 \%$ & $80.8 \%$ & $10.3 \%$ \\
\hline Colesterol total > 200 & $0.0 \%$ & $98.70 \%$ & $1.3 \%$ \\
\hline Colesterol LDL <130 & $0.0 \%$ & $96.2 \%$ & $3.8 \%$ \\
\hline Triglicéridos <200 & $0.0 \%$ & $97.4 \%$ & $2.6 \%$ \\
\hline Colesterol HDL & & & \\
Masculino > 30 & $40.0 \%$ & $60.0 \%$ & $0.0 \%$ \\
Femenino > 40 & $44.4 \%$ & $55.6 \%$ & $0.0 \%$ \\
\hline
\end{tabular}

Fuente: elaboración propia a partir de resultados de química sanguínea.

El $70 \%$ de los alumnos realizan un nivel de actividad física ligera predominando el sedentarismo, lejana de los estándares de estilos de vida saludable.

El rendimiento académico de los alumnos es bueno en un $77 \%$. En cuanto al nivel socioeconómico de los alumnos predomina el nivel medio en un $57 \%$, solo el $1 \%$ es alto y el resto es medio bajo.

En la tabla 4 se muestra que al relacionar el índice de masa corporal, balance energético, rendimiento académico, nivel de actividad de física y el nivel socioeconómico existe independencia entre las variables debido a que no se encontró diferencias estadísticamente significativas entre ellas. 
Tabla 4: Correlaciones de Pearson entre las variables

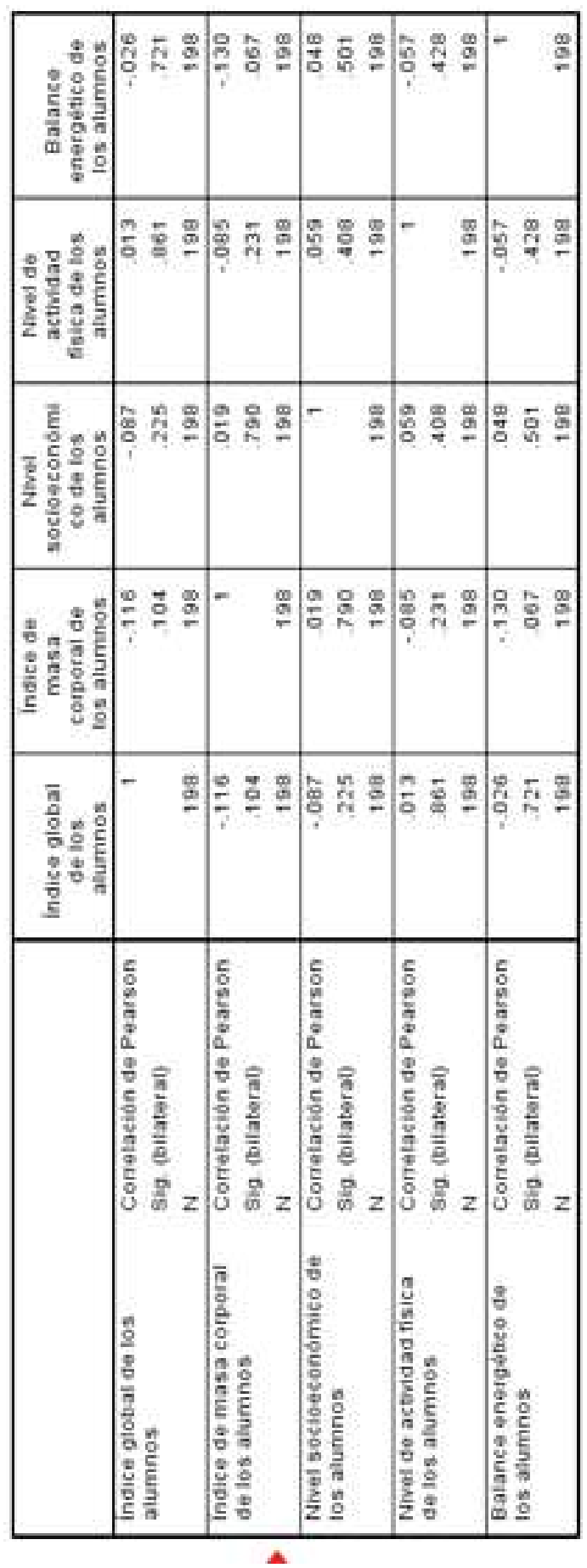

Fuente: elaboración propia a partir de resultados estadísticos 


\section{DISCUSIÓN}

El presente estudio mostró una prevalencia de sobrepeso de $20 \%$, de obesidad $7.6 \%$ y de normopeso $60 \%$ muy similar al estudio realizado en el Zamorano, Honduras en el 2012 con 315 estudiantes universitarios por Bayas Arévalo AV (Bayas Arévalo, A 2012) encontrándose sobrepeso en un $32.7 \%$, obesidad en $4.76 \%$ y normopeso en un $61.5 \%$. Al comparar los porcentajes de sobrepeso y de obesidad se cumple el dimorfismo de género que dice que las mujeres tienen mayor tendencia a la obesidad que los hombres (Carbajal, Azcona, A, 2013).

Por otro lado, el peso insuficiente obtenido es de en un $12.6 \%$ en la muestra, mientras que en el estudio realizado por Gallardo y Buen Abad (Gallardo Wong, I 2011) prevaleció en un $26.4 \%$, porcentajes apreciables, que llama la atención y puede ser tema de investigaciones futuras, con el fin de determinar si los universitarios están siendo afectados por trastornos de la conducta alimentaria, sobre todo, en las mujeres que generalmente se preocupan más de su imagen corporal.

Los resultados del presente estudio en cuanto a los hábitos alimentarios de universitarios revelan que tienen una dieta deficiente en energía, omiten el desayuno o si lo toman su ingesta calórica es deficiente, abusan de refrescos, una dieta deficiente en micronutrientes por su monotonía alimentaria, similares a los resultados obtenidos en el estudio realizado en universitarios en España el 2013 por Cervera (Cervera, Bunelf et 2013). Se reporta una dieta deficiente en cereales, legumbres, pescados, frutas y verduras al igual que en el estudio de Rodríguez, et al (Rodriguez, R.F, etal 2013) realizado en estudiantes universitarios en Chile el 2013 como un nivel de inactividad física alto, en donde los estudiantes masculinos son más activos que las estudiantes femeninas al igual que en el presente estudio. Similares resultados reporta el estudio de la población adulta de la Aldea El Jicarito, Honduras el 2012 de (Aguirre, Cando, 2012) donde incluye poca variabilidad de la dieta al igual que en este estudio.

En el presente estudio la mediana del consumo de energía fue de 1890 calorías (978-3611). La dieta en este estudio fue normal en límites para hidratos de carbono (56.63\%), alta en proteínas (17.44\%) e inferior en lípidos (18.61\%) y el $97.96 \%$ desayunan, muy similar resultado reporta el estudio de Gallardo y Buen Abad (2011) en México el 2010 en donde el consumo de energía fue de 1405 calorías (758-2900), la dieta fue normal en límites inferiores para hidratos de carbono (51.4\%), alta en proteínas $(19.3 \%)$ y normal en lípidos $(30.0 \%)$ y todos los estudiantes desayunaban. Excepto que solo el $22.64 \%$ de los alumnos eran sedentarios y en este estudio el 
$93.9 \%$ son sedentarios.

El porcentaje de los alumnos que presentan riesgo cardiovascular (33.3\%) fue menor que en las alumnas $(49.4 \%)$ de acuerdo al ICC una diferencia considerable entre ambos sexos, mientras que en el estudio realizado por Gallardo y Buen Abad8 con los estudiantes universitarios de Nutrición en el año 2010 reveló que el $28.3 \%$ de los alumnos presentaron riesgo, factor importante para padecer ECNT (Carbajal, Azcona, A, 2013).

Los resultados de este estudio fueron similares a los obtenidos en el estudio factores sociales y económicos asociados a la obesidad: los efectos de la inequidad y de la pobreza realizado por Álvarez-Castaño, et al 2012 se encontró que en relación con sus determinantes sociales y económicos, la obesidad es mayor en personas de estrato bajo y medio, en comparación con aquellas de estrato alto; afecta en mayor proporción a las personas con nivel de escolaridad primaria que a quienes tienen estudios secundarios. Estos últimos, a su vez, están más afectados que quienes tienen estudios universitarios. La probabilidad de padecer obesidad es mayor entre quienes viven en familias con ingresos menores que entre aquellos que devengan mayores ingresos. Estos datos hacen visible un gradiente social de la obesidad en relación con el nivel educativo, al estrato social y los ingresos familiares.

En el presente estudio no se encontró relación estadísticamente significativa entre el estado nutricional y el rendimiento académico de los alumnos como en el estudio de Bamba Espinoza (Bamba, Espinoza 2010) en Cuenca el 2010, sin embargo, en el estudio de Jiménez y Madrigal en México el 2009 que concluyó que los alumnos con sobrepeso y obesidad tienen un mayor índice de reprobación con respecto a los alumnos con normopeso y peso insuficiente, interesante estudio a realizar con esta población a futuro.

\section{CONCLUSIONES}

Los resultados no pueden ser generalizados, sin embargo, dan una visión sobre la tendencia de los factores de riesgo, composición corporal, hábitos alimentarios, la ingesta hídrica y la actividad física. Más de la mitad de los alumnos participantes tiene buen estado nutricional de acuerdo al IMC ya que tienen normopeso. Con base en el IMC de los universitarios se observa que en ambos sexos presentan los patrones de dimorfismo sexual y hay una predisposición al exceso de grasa corporal en ambos 
sexos, obteniéndose sobre-peso en los hombres y obesidad en las mujeres. Los alumnos (hombres) presentan menos riesgo que las alumnas (mujeres) de padecer ECNT según los datos obtenidos de la relación cintura-cadera. En cuanto al porcentaje de grasa corporal se encontró valores de ésta menores a lo recomendado en ambos sexos, pero las alumnas presentaron porcentajes más altos que los hombres, cumpliéndose la tendencia del sexo femenino hacia la obesidad y del masculino al sobrepeso.

Más de la mitad de la muestra se ubicó en el nivel socioeconómico medio, en cuyas familias el ingreso familiar solo cubre los gastos básicos y el nivel de escolaridad de las madres en su mayoría es de secundaria por lo cual no hay suficiente educación nutricional para que en los hogares se administre una dieta equilibrada que permita tener una densidad alta de nutrientes. El nivel socioeconómico bajo presentó una mayor prevalencia de sobrepeso y obesidad. Sin embargo, es necesario continuar con la tarea de investigar los estados de mala nutrición en la población universitaria ya sea por defecto o por exceso, así reconocer factores de riesgo, dar juicios acertados a tiempo, con el propósito de impulsar actividades preventivas que impidan el aumento de enfermedades crónicas no trasmisibles o complicaciones de las mismas ya que ellos serán la población económicamente activa. En las futuras investigaciones sobre el estado nutricional de los alumnos universitarios hay que incluir la evaluación bioquímica, dietética y clínica para tener un panorama más completo e involucrar a todos los miembros del equipo de salud en la comunidad universitaria como médicos, nutricionistas, microbiólogos y farmacéuticos.

La dieta de los alumnos no es equilibrada, presenta un balance energético negativo, el patrón alimentario es monótono, caracterizado por bajo consumo de frutas, verduras, pescado, por ende, resulta en una dieta con baja densidad nutritiva. La ingesta hídrica dista de las ingestas recomendadas en la mayoría de los alumnos y predomina más el sedentarismo en las mujeres que en los varones. La ingesta de alimentos se desvía de las recomendaciones del INCAP, presentándose una baja ingesta calórica y en cuanto a la distribución calórica de macronutrientes es alta en proteína, alta en carbohidratos y baja en grasas totales. La distribución calórica es menor a la recomendada en el desayuno y en las meriendas y mayor a lo recomendado en el almuerzo y la cena, de esta manera se favorece el sobrepeso. Un factor positivo a resaltar es que realizan en su mayoría 3 a 4 comidas recomendadas, aunque no se cumpla su distribución calórica. Preocupa por el hecho de que la efectividad del trabajo intelectual depende de lo que hemos consumido en la alimentación, así desayunos nutricionalmente incorrectos se asocian a un menor rendimiento físico e intelectual, a 
aumentar los desequilibrios y a alcanzar las ingestas recomendadas de energía y nutrientes.

Este estudio brinda por primera vez datos de una muestra de la facultad de Ciencias Químicas y Farmacia, concluyéndose que no se encontró relación entre el estado nutricional, la actividad física, el nivel socioeconómico y el rendimiento académico.

\section{RECOMENDACIONES}

- Establecer alternativas alimentarias en la universidad que sean saludables y de fácil acceso para los alumnos.

- Monitorear individualmente el estado nutricional de los alumnos que presentaron factores de riesgo de acuerdo a su estado nutricional como de su rendimiento académico.

- Recomendar la ingesta de suplementos o alimentos fortificados en especial a los alumnos que presentaron subadecuación, en vista que se presentó una dieta monótona resultando difícil que se estén aportando todos los nutrientes.

- La Facultad de Química y Farmacia debe contar con un espacio disponible para la práctica de actividad física, donde se puedan realizar diferentes actividades, en diferentes horarios evitando el inconveniente del tiempo invertido en el traslado a otro lugar y permitiendo su realización a la hora que el alumno tenga un tiempo libre. Además, se debe promover la práctica de actividad física mostrando sus beneficios y enseñar a los alumnos a gestionar el tiempo en sus horas de descanso.

- En este estudio se determinó el porcentaje de calorías correspondiente al consumo de grasas totales obteniéndose un valor menor a lo recomendado. Sería recomendable realizar posteriormente un estudio para determinar los ácidos grasos mono insaturados (AGM), ácidos grasos polinsaturados (AGP), ácidos grasos saturados (AGS) y colesterol por el alto consumo de manteca (AGS) y margarinas (AGS) en la preparación de los alimentos en nuestro país ya que surge la inquietud de que el porcentaje de grasas totales sean saturadas.

\section{AGRADECIMIENTOS}

Agradecimientos especiales por el apoyo del personal docente y autoridades de la 
Carrera de Química y Farmacia, de la Carrera de Nutrición, del Programa de Salud de la Vicerrectoría de Orientación y Asuntos Estudiantiles de la UNAH y por su aporte económico de la Dirección de Investigación Científica y Postgrado para la realización de este estudio.

\section{REFERENCIAS BIBLIOGRÁFICAS}

OMS. (2013). WHO. Recuperado de WHO: www.who.int./features/factfiles/obesity/es/

OMS. (2009). WHO. Recuperado de WHO: http://www.who.int/social_determinants/thecommission/finalreport/key_concepts/es/.

Carbajal Azcona, A. Conceptos Básicos. En Carbajal Azcona A. Manual de nutrición y dietética. Madrid: Universidad Complutense de Madrid; 2013. p. 6-9 . Recuperado de: https://www.ucm.es/data/cont/docs/458-2013-08-19-Manual-nutr-diet-indice.pdf

Carbajal Azcona A. (2013). Manual de nutrición y dietética. Dieta equilibrida. Madrid, España. Recuperado de https://www.ucm.es/data/.../458-2013-08-19-Manual-nutr-diet-indice.pdf Rodriguez, R.F., et-al. Hábitos alimentarios, actividad física y nivel socioeconómico en estudiantes universitarios de Chile. Nutr Hosp., 28(2), p.447-455. Recuperado de : http://www.scielo.isciii.es/scielo.php

Álvarez Castaño, L.S.; Goez Rueda ,J.D.; Carreño Aguirre ,C. (2012). Factores sociales y económicos asociados a la obesidad. Rev. Gerenc Polit, 11(23), p.98-110. Recuperado de http://www.scielo.org.co/pdf/rgps/v11n23/v11n23a06.pdf

Córdoba, D., Carmona, M., Terán, O. (2013). Relación del estilo de vida y estado de nutrición en estudiantes universitarios. Medwave. Recuperado de: http://www.medwave.cl/link.cgi/medwave/estudios/investigacion

Gallardo Wong I, B. A. (2011). Mala Nutrición en estudiantes universitarios de la escuela dietética y nutrición del ISSSTE. Revista Médica UV, 11(1), p.6-11. Recuperado de: https://www.uv.mx/rm/num_anteriores/revmedica_vol11_num1/articulos/mala.pdf

Bamba Espinoza, E. S. (2010). Prevalencia de la mala nutrición y su relación con el rendimiento académico en los niños de la escuela "Julio Montovelle". Universidad de Cuenca. Recuperado de http://www.dspace.ucuenca.edu.ec/jspui/bitstream/123456789/3516/1 IMED22.pdf

Rivera M F., I.C. (2010). Conductas alimentarias y actividad física en niños escolares de Tegucigalpa. Revista Médica Hondureña. Honduras; 78(2), p.65-69. Recuperado de: http://www.bvs.hn/RMH/pdf/2010/pdf/Vol78-2-2010-4.pdf

Castro Amador A, M. M. (2000). Valoración nutricional intrahospitalaria en niños del Hospital Materno Infantil e Instituto Hondureño de Seguridad Social. Revista Médica Hondureña. 5 (3), p. 211-217. Disponible en http://www.bvs.hn/RMP/pdf/2000/pdf/Vol5-3-2000-4.pdf Aguirre Cando ME. (2012). Evaluación de consumo de alimentos de la población adulta de la Aldea El Jicarito, Francisco Morazán. Honduras. Escuela Agrícola Panamericana . 
Recuperado de: https://bdigital.zamorano.edu/bitstream/11036/950/1/AGI-02012-T001 Esenarro Aguilar, et-al. (2012). Guía técnica para la valoración nutricional antropométrica de la persona adulta. Perú. Obtenido de Instituto Nacional de Salud:http://www.ins.gob.pe/repositorioaps/0/5/jer/otros_lamejo_cenan/GuiatecnicaAdultoMayor.pdf

Arias Fidias, G. (2012). El proyecto de investigación. Introduccióna la metodología científica. p.85 6ta ed. República Bolivariana de Venezuela. Editorial Episteme. Disponible en https://ebevidencia.com/wp-content/uploads/2014/12/EL-PROYECTO-DE-INVESTIGACION-6ta-Ed.-FIDIAS-G.-ARIAS.pdf

Mataix Verdú J, López Jurado Romero de la Cruz M. (2009). Valoración de estado nutricional. Estructura y composición corporal. En Mataix Verdú J, editor. Nutrición y alimentación. Vol. 2. Barcelona: OCÉANO/ergon; p.1004-1025.

Carbajal Azcona A. (2013) Composición corporal. En Carbajal Azcona, A. Manual de nutrición y dietética. Madrid. Universidad Complutense;p.11-17. Disponible en:https://www.ucm.es/data/cont/docs/458-2013-08-19-Manual-nutr-diet-indice.pdf

Menchú M Torun B, E. L. (2012). Recomendaciones dietéticas diarias del INCAP. Energía y macronutrientes. INCAP, p.21-81. Disponible en http://www.incap.org.gt/ciipec/index.php/es/ciipec/noticias/565-recomendaciones-dieteticas-diarias-del-incap-2

Bayas Arévalo A. (2012). Evaluación del estado nutricional de los estudiantes de la Escuela Agrícola Panamericana. (Zamorano, Ed.) bdigital. Recuperado de: https://bdigital.zamorano.edu/bitstream/11036/871/3/AGI-2012-T005.pdf

Cervera Buriel F, et-al (2013) Hábitos alimentarios y evaluación nutricional en una población universitaria. Nutrición Hospitalaria,28(2) p.438-446. Obtenido de http://scielo.isciii.es/p$\mathrm{df} / \mathrm{nh} / \mathrm{v} 28 \mathrm{n} 2 / 230$ riginal18.pdf

Jiménez Guerrero M, Madrigal Fritsch H. (2009). Evaluación del Estado Nutricional de los alumnos de la Escuela Prepatoria de ULSA. Redalyc, 8(31), 35-48. Recuperado de http://www.redalyc.org/ 